\title{
The Role of Contrast Enhanced Digital Mammography in Evaluation of Breast Masses
}

\author{
Hayam A. Abdellatif, Shrouk M. El Dalatony, ElSayed E. ElSayed \\ Department of Radiodiagnosis, Faculty of Medicine - Menofia University, Egypt \\ *Corresponding Author: Shrouk M El Dalatony, Mobile: (+20) 01090343620, Email: shrouk.roka.sh@ gmail.com
}

\begin{abstract}
Background: A thorough clinical breast examination, imaging, and tissue sampling are needed for a definitive diagnosis of breast diseases. Contrast-enhanced digital mammography (CEDM) is the second type of advanced technology stemming from the digital platform (the first being tomosynthesis) that proved to be promising in this field. Objectives: The aim of this study was to evaluate the role of contrast enhanced digital mammography in diagnosis of breast masses. Patients and Methods: The study included 30 participants who visited the hospital for assessing their complaints of breast masses during the period from May 2019 to May 2020 after taking their consent. Results: The contrast enhanced digital mammography (CEDM) proved that 17 patients $(56.7 \%)$ proved with malignant masses, $10(33.3 \%)$ benign and $3(10 \%)$ normal that was confirmed by final diagnosis of these cases. The sensitivity and specificity of the CEDM $(100 \%, 92.86 \%)$ respectively, that expresses high levels of both parameters. Conclusion: CEDM has higher diagnostic value that can enhance the process of early cancer diagnosis, postoperative follow up, and treatment plans.
\end{abstract}

Key words: Breast masses, CEDM, Mammography, Sensitivity, Specificity.

\section{INTRODUCTION}

Breast masses are common and usually benign, but efficient evaluation and prompt diagnosis are necessary to rule out malignancy. A thorough clinical breast examination, imaging, and tissue sampling are needed for a definitive diagnosis. Breast masses have a variety of etiologies, benign and malignant ${ }^{(\mathbf{1})}$. Although the vast majority of the lesions that occur in the breast are benign, much concern is given to malignant lesions of the breast because breast cancer is the most common malignancy in women in western countries ${ }^{(2)}$. Benign breast masses include fibroadenoma (FA), fibrocystic change (FCC), benign phyllodes tumour (BPT), papilloma (PL) and inflammation (IF). Malignant masses include invasive ductal carcinoma (IDC), ductal carcinoma in situ (DCIS), invasive lobular carcinoma (ILC), invasive papillary carcinoma (IPC) and mucinous carcinoma (MC) ${ }^{(3)}$.

Mammography remains the method of choice for breast imaging, despite the development and improvement of other imaging modalities in recent decades. In the field of mammography too, significant technical improvements were realized, mainly owing to the introduction of digital mammography. However, even though the diagnostic accuracy of full field digital mammography (FFDM) is good, it depends heavily on breast density ${ }^{(4)}$.

Potential use of contrast-enhanced digital mammography (CEDM) for supplemental screening may benefit women with an intermediate to high lifetime risk of breast cancer, including women with dense breast tissue. Initial studies suggest that, for dense breast tissue, CEDM performs better than 2D mammography for detecting malignancy and avoiding false negative results ${ }^{(\mathbf{5})}$. Contrast-enhanced digital mammography (CEDM) is the second type of advanced technology stemming from the digital platform (the first being tomosynthesis). The success of mammography in breast cancer detection stems from its ability to detect morphologic abnormalities ${ }^{\left({ }^{(}\right)}$.

Contrast-enhanced digital mammography is a dual-energy technique performed after the IV administration of iodinated contrast agent to identify abnormalities based on angiogenesis, as well as morphologic features and density, resulting in improved breast cancer detection. The physiologic information provided by CEDM is similar to that provided by breast MRI, without the added time or expense of conventional breast MRI protocols ${ }^{(7)}$.

The combination of morphologic and physiologic information provided by CEM has been shown to provide superior sensitivity and specificity in diagnosing breast cancer when compared with digital mammography alone ${ }^{(8)}$. Results of clinical studies show that CEDM is significantly more sensitive and specific than mammography alone and has sensitivity and specificity comparable to contrast-enhanced breast MRI ${ }^{(9)}$.

The aim of this study was to evaluate the role of contrast enhanced digital mammography in diagnosis of breast masses.

\section{SUBJECTS AND METHODS}

The study was conducted in the period between May 2019 and May 2020. It included 30 cases. Cases were collected from Al-Agouza Police Hospital Radiology department.

The Inclusion criteria of the study subjects were patients with increased risk of developing breast cancer (moderate or high risk) who have at least single sonomammographic suspicious lesions for better characterization. 
- BIRADS 4 and 5, i.e. mass with indistinct or speculated margins and irregular shape on sonomammography, micro-calcifications of suspicious morphology, architecture distortion, focal asymmetry and complex breast cysts.

- Patients with axillary lymphadenopathy with no sono-mammographic detected breast lesions.

- Patients with pathologically proved breast cancer for further staging (BIRADS 6).

- High-risk patient with dense breast.

While the following subjects were excluded from the study:

- Patients with average risk of developing breast cancer.

- Contraindication to mammography, e.g. pregnant women

- Contraindication to IV contrast, e.g. patients with renal impairment, allergic patients or those known to have history of anaphylactic reaction from contrast media.

\section{All patients were submitted to the following:}

1. Personal interview for socio-demographic and clinical data collection: Including patient's name, age, marital status and number of offspring's, residence and phone number, past history and family history, diagnosis and duration of illness.

2. Imaging procedure: All patients underwent breast digital mammography and contrast enhanced spectral mammography followed by ultrasound.

a. Equipment: (i) The ultrasound examination was performed using GE ultrasound scanner (GE) linear array electronic probe. (ii) Mammographic examination was performed using Senographe Essential, GE healthcare Full Field Digital Mammography machine that was upgraded to provide CESM. (iii) Senographe Essential has a dual anode (Rhodium Molybdenum) with cesium iodide (CsI) digital detector.

b. Image analysis and interpretation of sonomammography:

Breast density was assessed for each patient according to ACR Mammography BIRADS atlas 2013; ACR A (the breasts are almost entirely fatty), ACR B (scattered fibro-glandular tissue), ACR C (The breasts are heterogeneously dense) and ACR D (The breasts are extremely dense). For mammography, each lesion was evaluated regarding the site, type (mass, architectural distortion, asymmetry, calcifications). For ultrasound, each lesion was evaluated whether mass, simple cyst, complicated cyst or area of parenchyma distortion. Axillary lymph nodes were evaluated by ultrasound. We determined the sonomammographic BIRADS category of each lesion according to the BIRADS atlas 2018, guided by the results of clinical data and sono-mammographic findings but blind to final pathologic diagnosis.

c. Image analysis and interpretation of contrast enhanced spectral mammography:

Level of background parenchymal enhancement was assessed in each patient (minimal, mild, moderate and marked). CESM MLO and CC views were assessed for the presence or absence of enhancing lesions. Enhancing lesions were then classified as mass or non-mass. When an enhancing mass lesion was detected, it was further assessed for its margins (circumscribed, not circumscribed irregular or not circumscribed speculated), degree of enhancement (mild, moderate and severe) and pattern of internal enhancement (homogenous, heterogeneous, septations or ring enhancement). When nonenhancing mass lesion was detected, it was further assessed for distribution (focal, linear, segmental, regional, multiregional or diffuse), and pattern of internal enhancement (homogenous, heterogeneous, clustered and clumped) and degree of enhancement (mild, moderate and severe). Axillary lymph nodes were assessed. We determined the BIRADS category of each lesion in reference to MRI BIRADS atlas 2018 morphology descriptors as there is no standardized BIRADS Lexicon to CESM, guided by the results of clinical data and CESM findings but blinded to the final pathological diagnosis.

\section{Ethical consent:}

An approval of the study was obtained from Menofia University Academic and Ethical Committee. Every patient signed an informed written consent for acceptance of the operation.

\section{Statistical Methods}

Results were collected, tabulated and statistically analyzed by an IBM personal computer with SPSS (Statistical Package for the Social Sciences) version 25 software. Qualitative data were presented as frequency and percentage and were compared by Fisher's exact test. A P-value of $<0.05$ was considered statistically significant.

\section{RESULTS}

Figure (1) shows that $56.7 \%$ of the studied groups were in the age group of 40-59. 


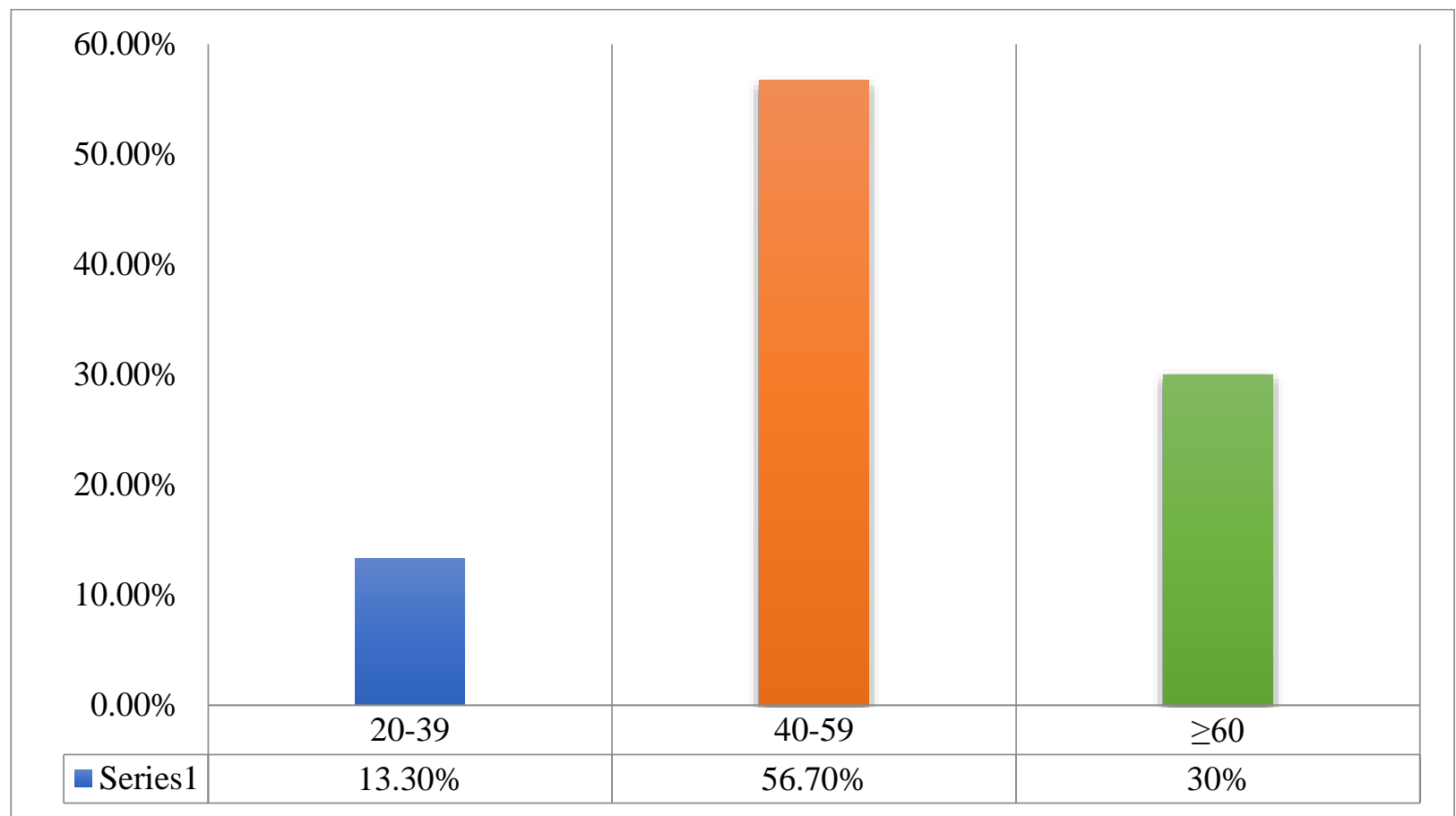

Fig. (1): Frequency distribution of the studied group according to their age category.

Figure 2 shows that $66.7 \%$ of the study participants had malignant lesions according to the final mammography BIRADS.

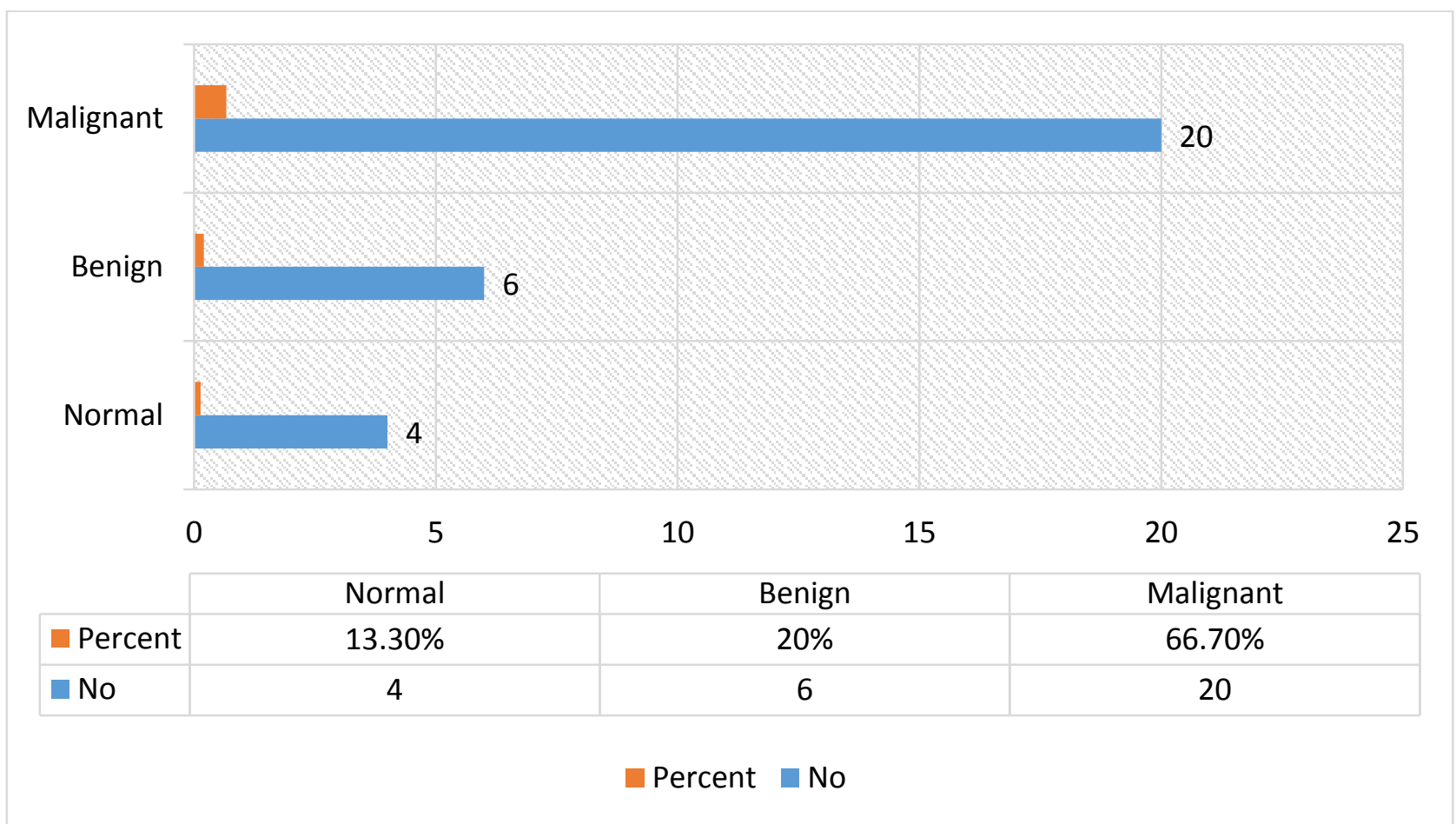

Fig. (2): Frequency distribution of the studied group according to the final mammography BIRADS.

According to CEDM BIRADS $56.7 \%$ of patients presented with malignant lesions (Fig. 3). 


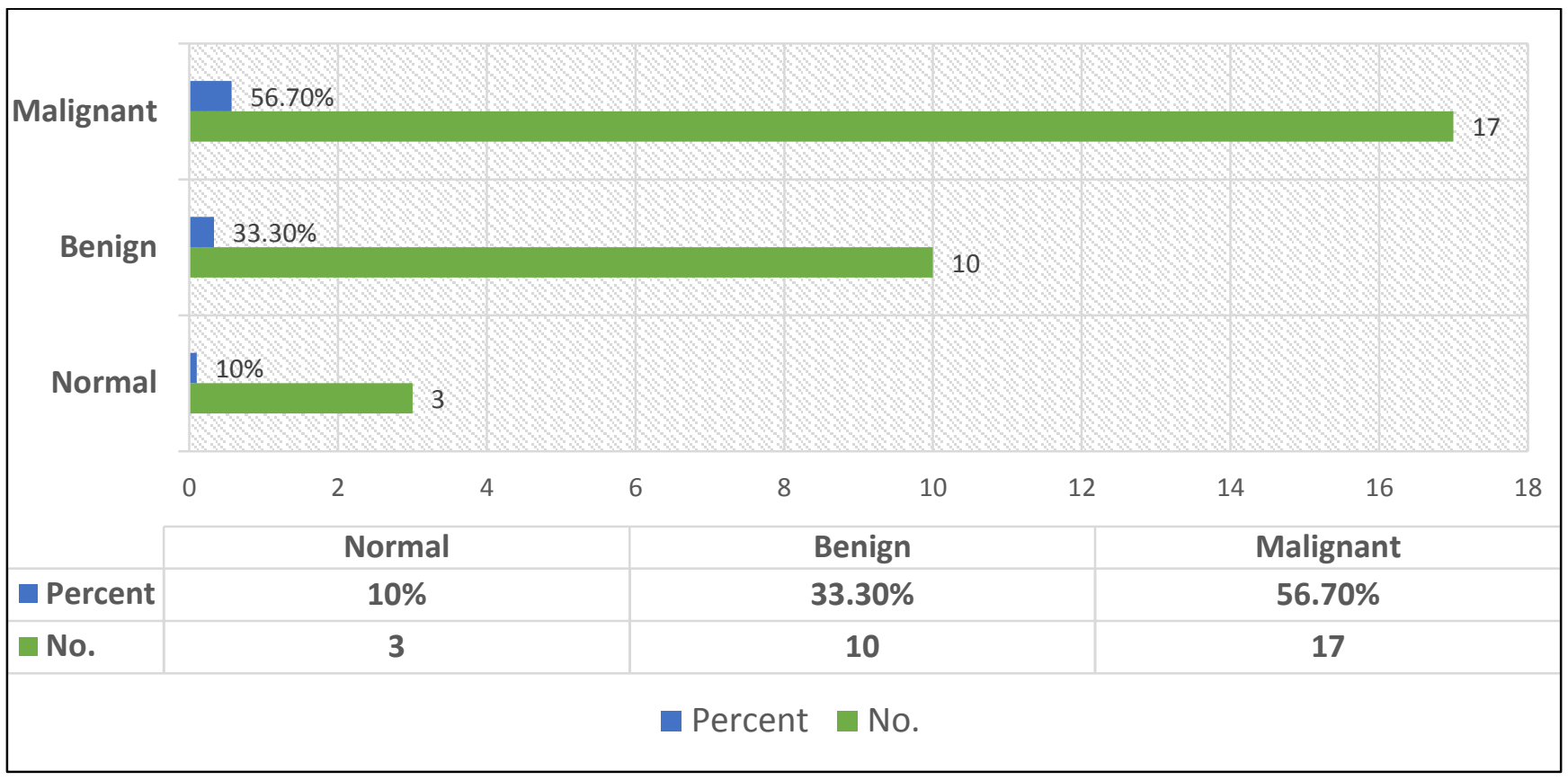

Fig. (3): Frequency distribution of the studied group according to the CEDM BIRADS.

This figure (4) shows that according to the final diagnosis $56.7 \%$ were malignant.

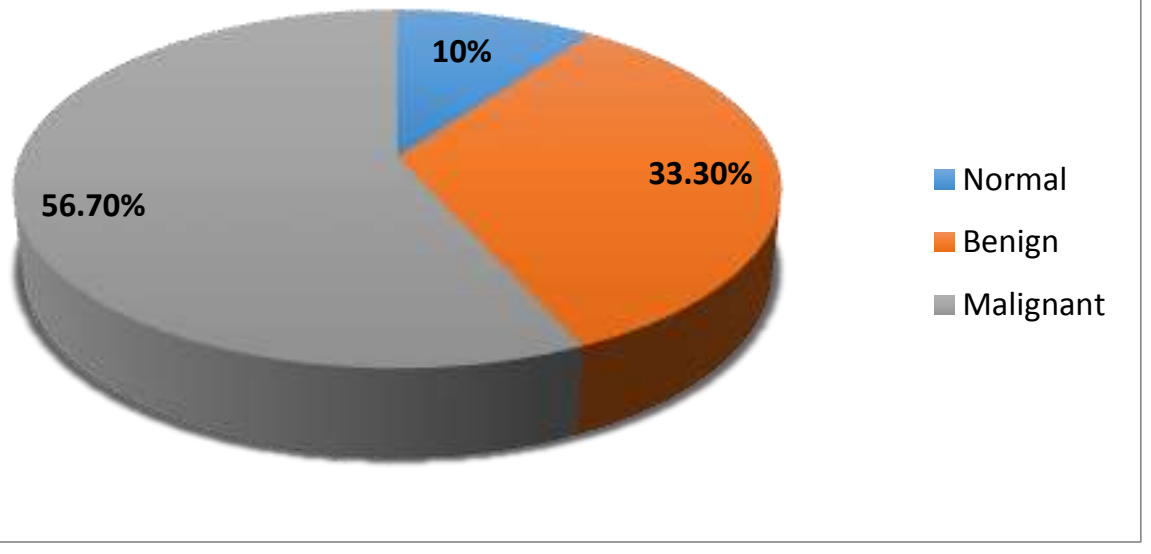

Fig. (4): Frequency distribution of the studied group according to their final diagnosis.

There was a highly significant association between mammography and CEDM end diagnosis (Table 1).

Table (1): Comparison between mammography and CEDM final diagnosis results.

\begin{tabular}{|c|c|c|c|c|c|c|c|c|}
\hline Variable & \multicolumn{6}{|c|}{ CEDM } & Test of significance & \multirow{3}{*}{$\begin{array}{c}\text { P- } \\
\text { Value }\end{array}$} \\
\hline Mammography & \multicolumn{2}{|c|}{ Normal } & \multicolumn{2}{|c|}{ Benign } & \multicolumn{2}{|c|}{ Malignant } & & \\
\hline Diagnosis & No & $\%$ & No & $\%$ & No & $\%$ & Fisher's exact & \\
\hline Normal & 2 & 66.67 & 3 & 30 & 0 & 0.00 & \multirow{4}{*}{24.36} & \multirow{4}{*}{$<0.001^{*}$} \\
\hline Benign & 0 & 0.00 & 5 & 50 & 0 & 0.00 & & \\
\hline Malignant & 1 & 33.33 & 2 & 20 & 17 & 100 & & \\
\hline Total & 3 & 100 & 10 & 100 & 17 & 100 & & \\
\hline
\end{tabular}

The sensitivity and specificity of the CEDM $(100 \%, 92.86 \%)$ respectively, are displayed in table 2, which proves high levels of both parameters.

Table (2): Sensitivity, specificity, $+\mathrm{PV} *,-\mathrm{PV} * *$, accuracy and AUC*** of CEDM.

\begin{tabular}{|c|c|c|c|c|c|c|c|}
\hline & $\begin{array}{c}\text { Cut off } \\
\text { point }\end{array}$ & Sensitivity & Specificity & +PV & -NPV & Accuracy & AUC \\
\hline CEDM & $>3$ & $100.0 \%$ & $92.86 \%$ & $100.0 \%$ & $94.1 \%$ & $100.0 \%$ & $98.9 \%$ \\
\hline
\end{tabular}

*Positive Predictive Value, ${ }^{* *}$ Negative Predictive Value, ${ }^{* * *}$ Area under the Curve 


\section{CASE 1}

Comment: CESM disproved the presence of suspicious lesion seen in mammography.

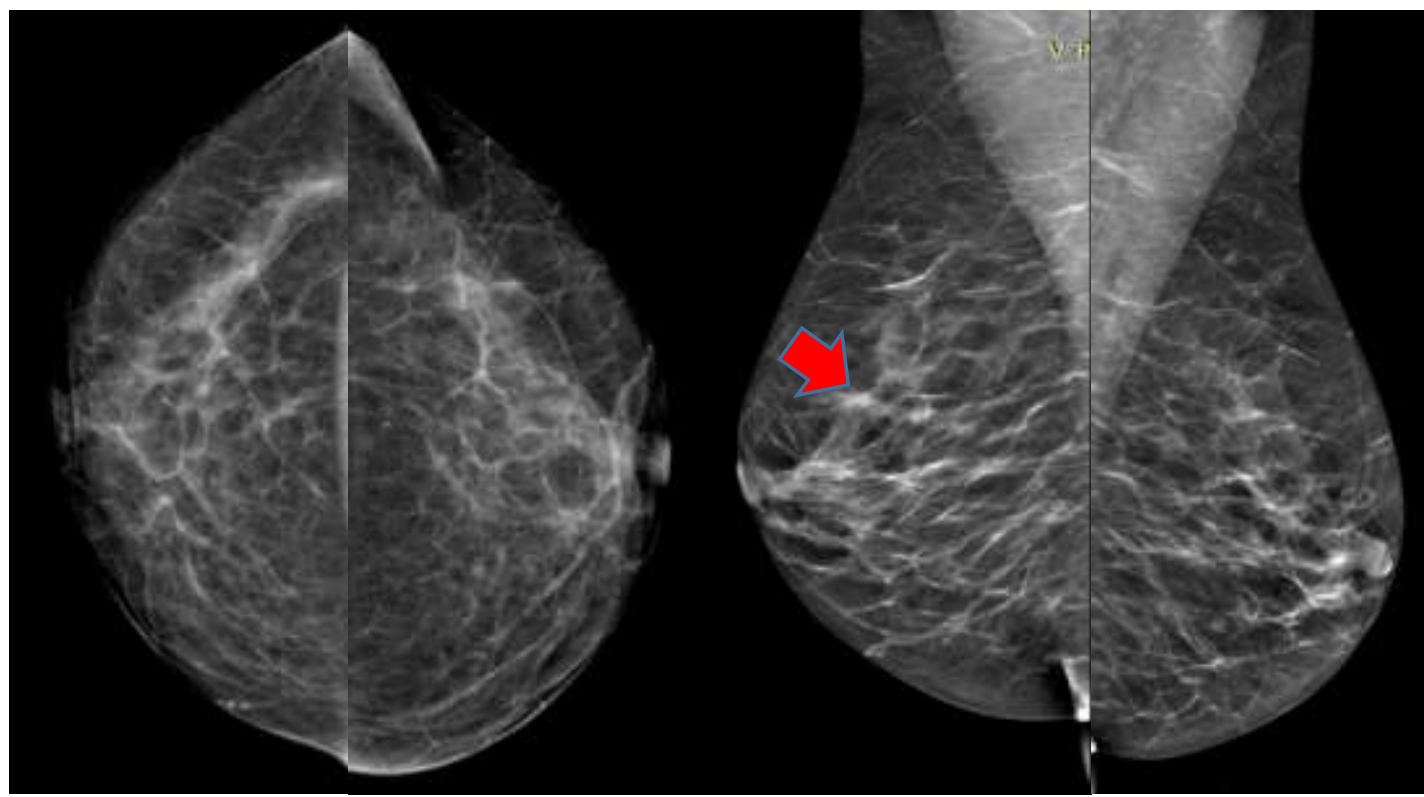

Fig. (5): A) FFD mammography (CC \& MLO views).

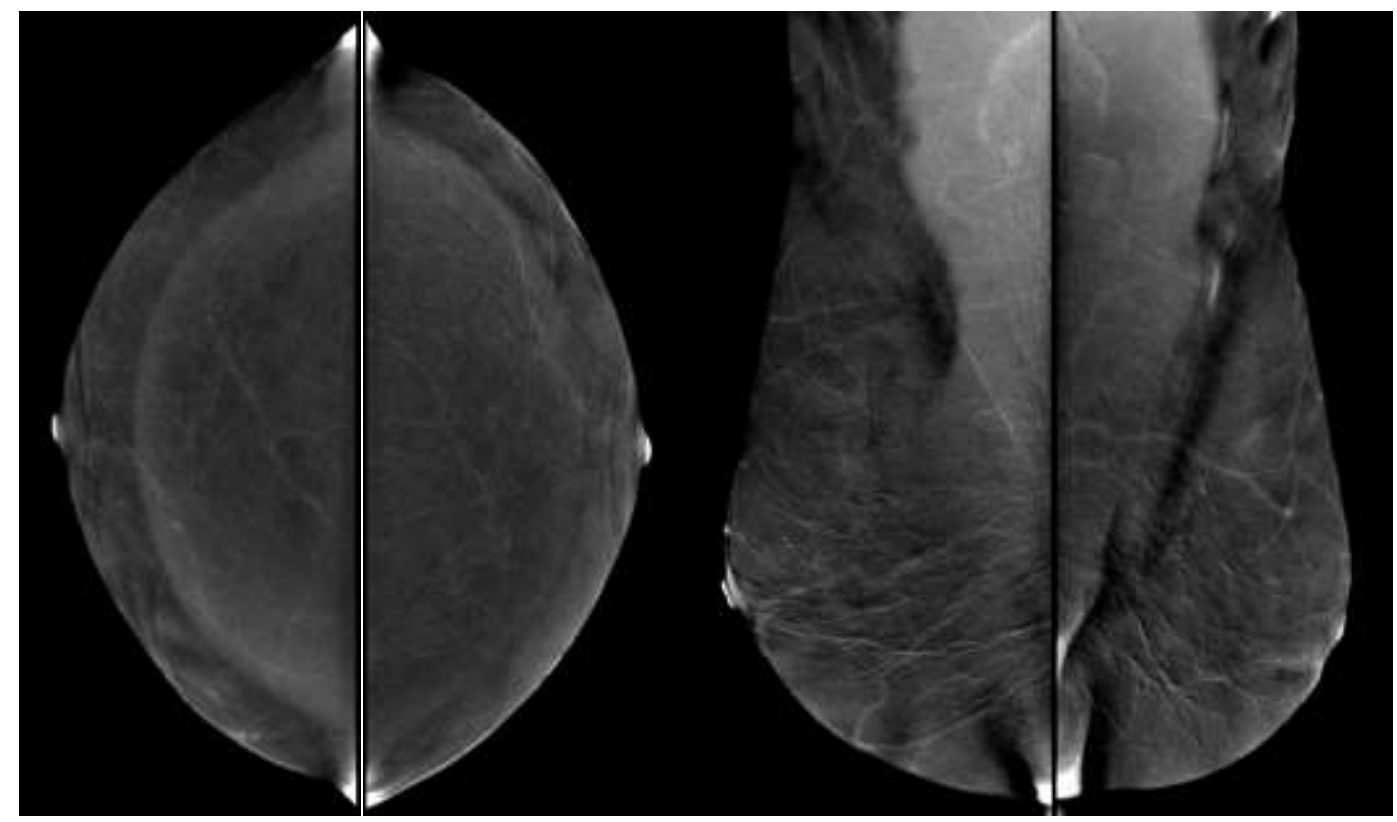

Fig. (5): B) Contrast enhanced spectral Mammography (CC \& MLO views). 


\section{CASE 2}

Comment: CESM proved that the lesion is malignant in nature and demonstrated that it is a multifocal breast lesion.

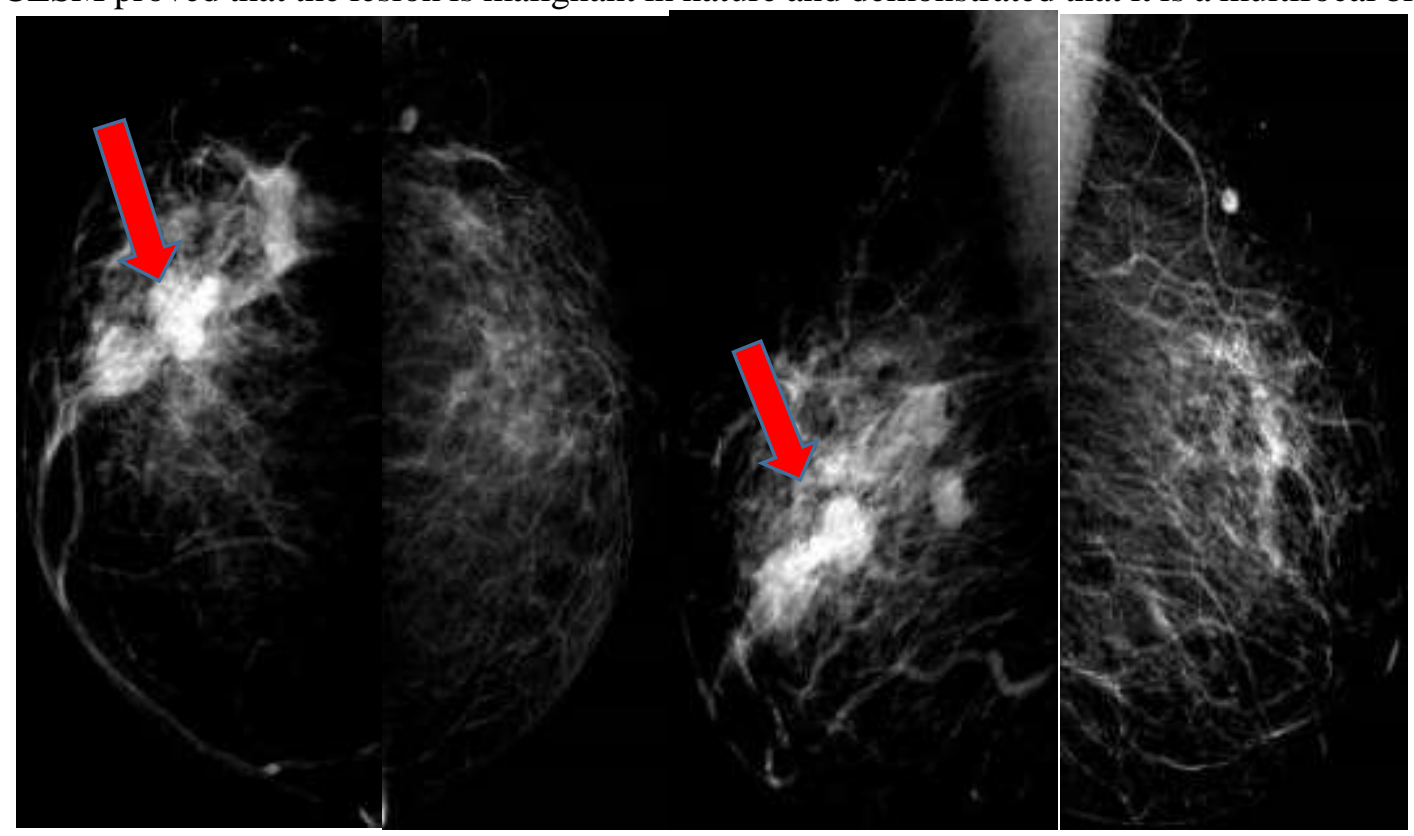

Fig. (6): A) FFD mammography (CC \& MLO views).
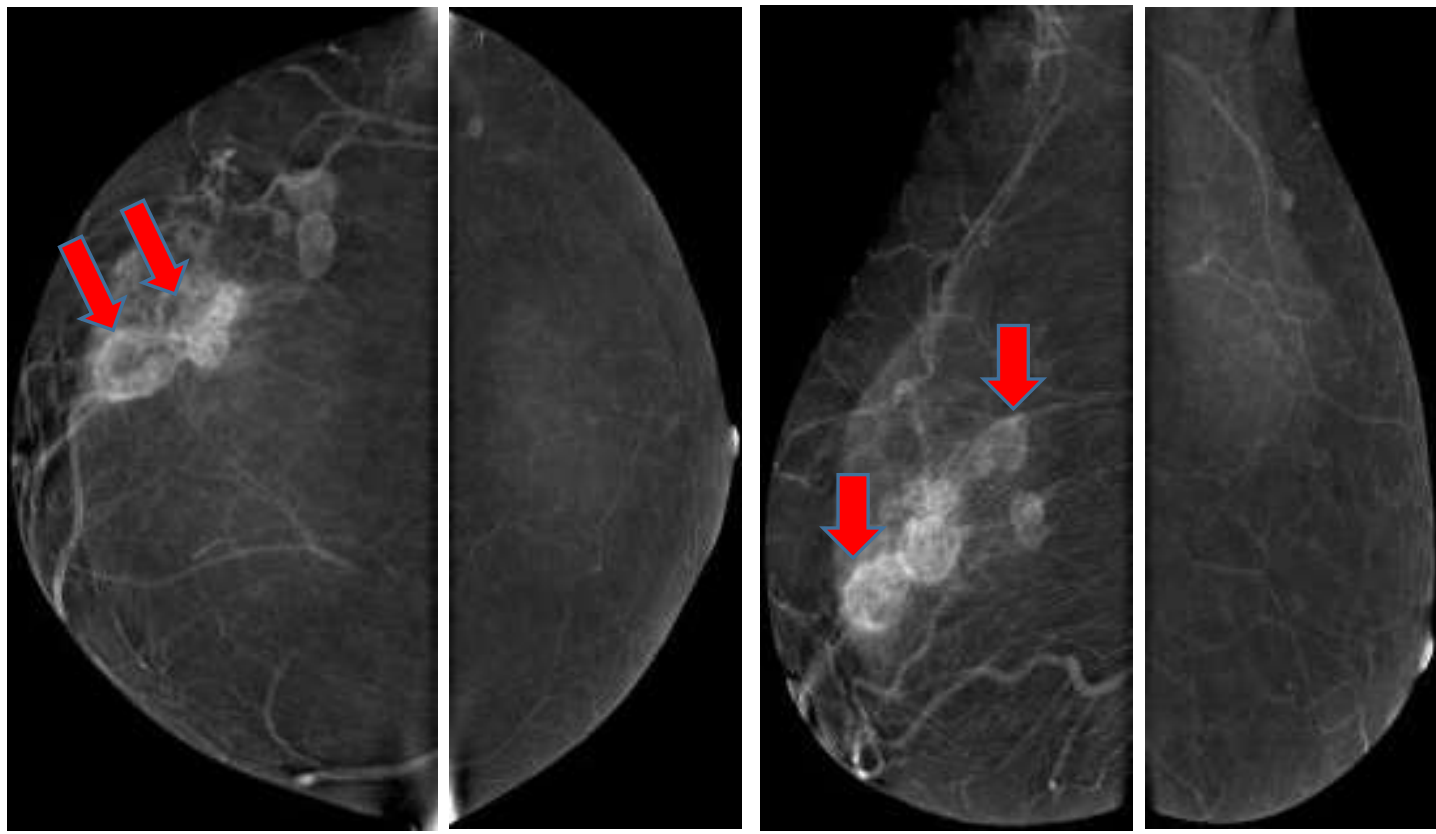

Fig. (6): B) Contrast enhanced spectral Mammography (CC \& MLO views). 


\section{CASE 3}

Comment: CESM proved the benign nature of breast lesions (Bilateral extensive mammary adenosis).

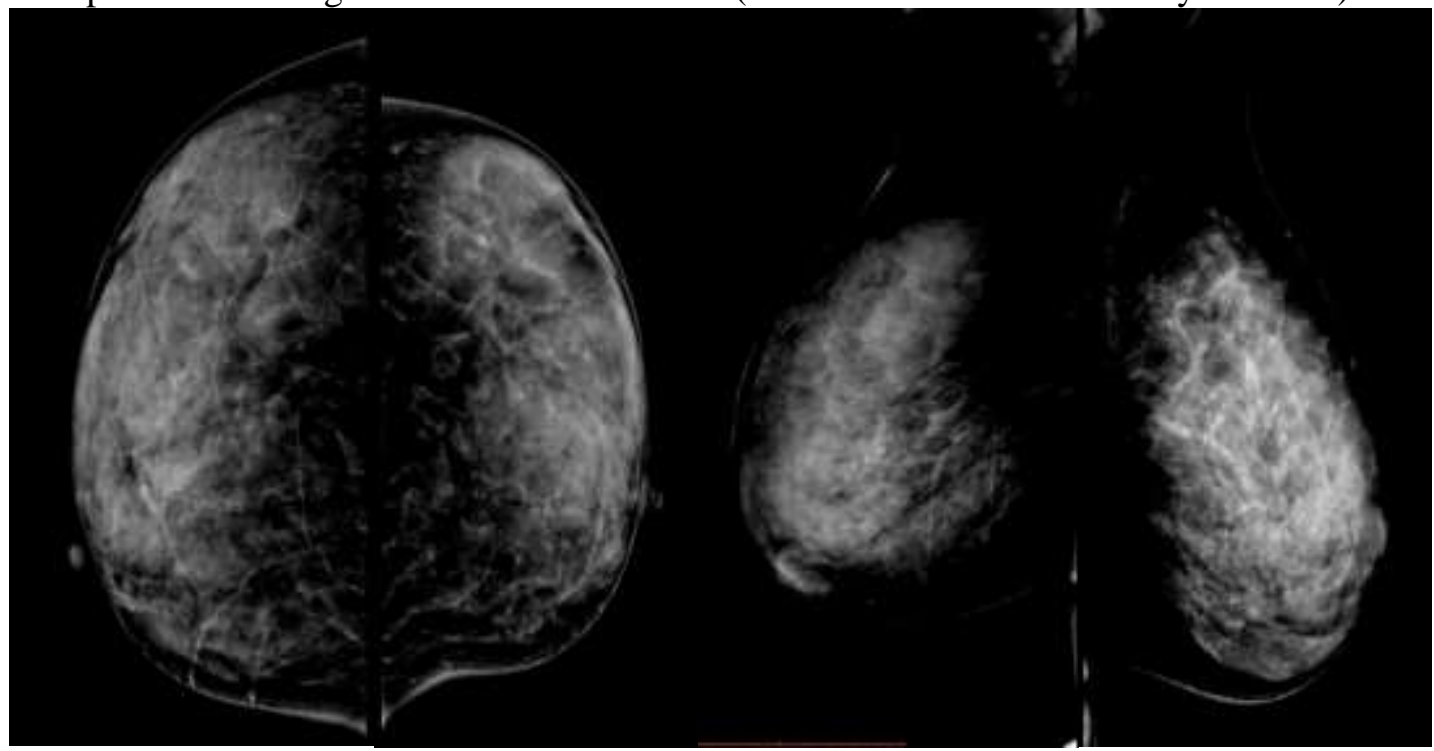

Fig. (7): A) FFD mammography (CC \& MLO views).

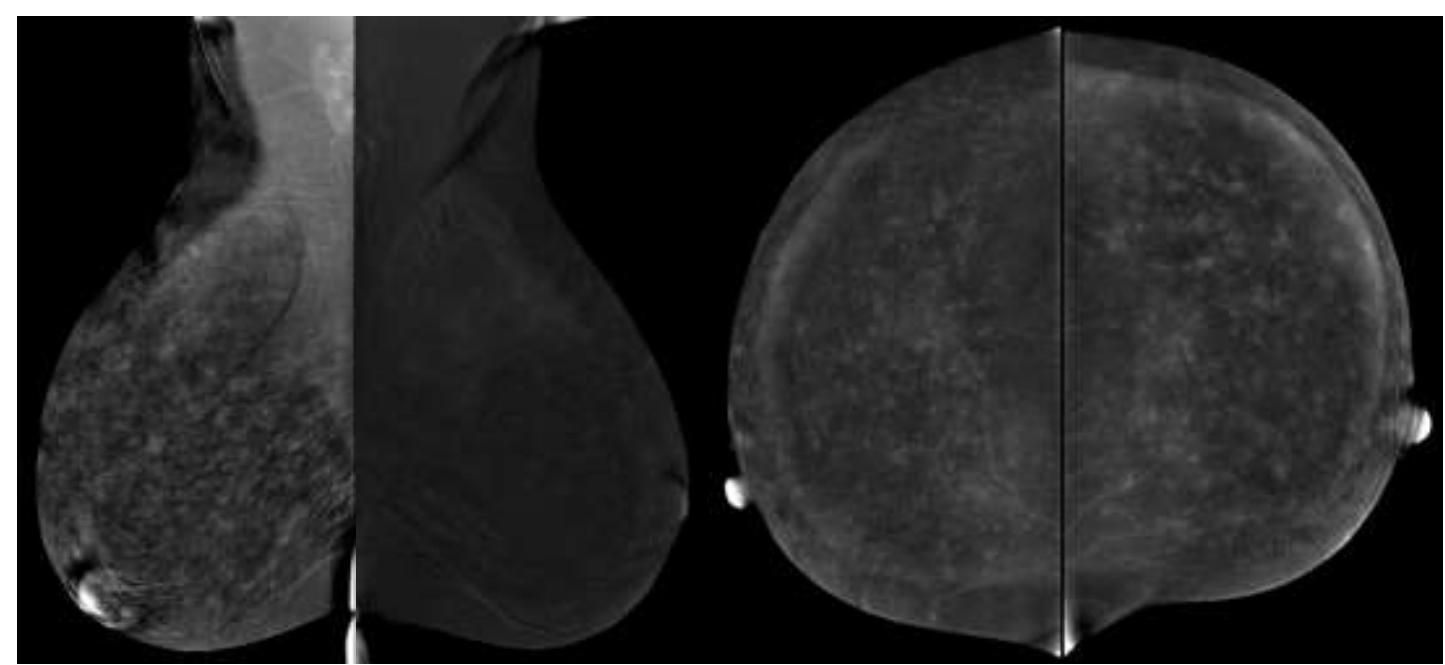

Fig. (7): B) Contrast enhanced spectral Mammography (CC \& MLO views). 


\section{CASE 4}

Comment: CESM showed an underlying lesion not seen clearly on mammography and proved its malignant nature (Multifocal malignant mass with intra-ductal extension and pathological axillary LNs).

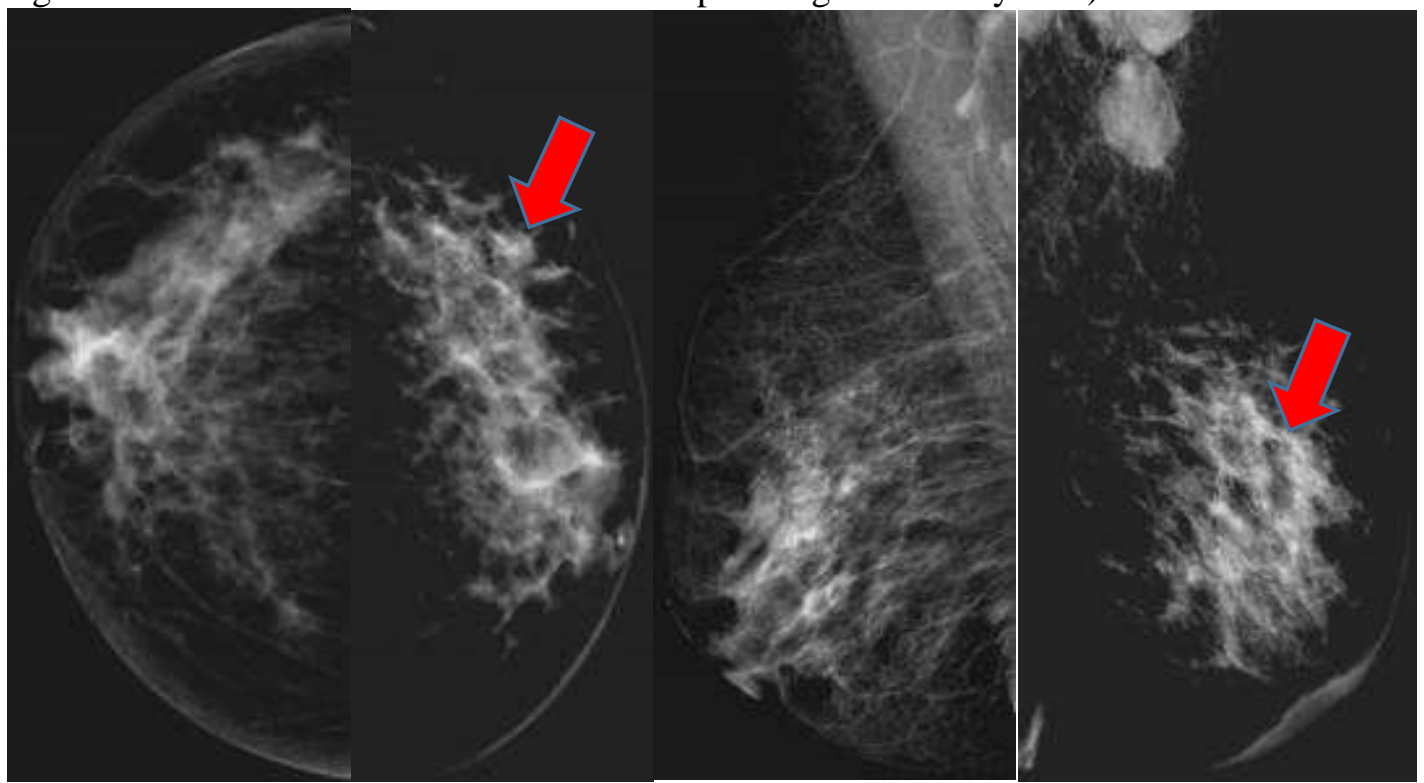

Fig. (8): A) FFD mammography (CC \& MLO views).
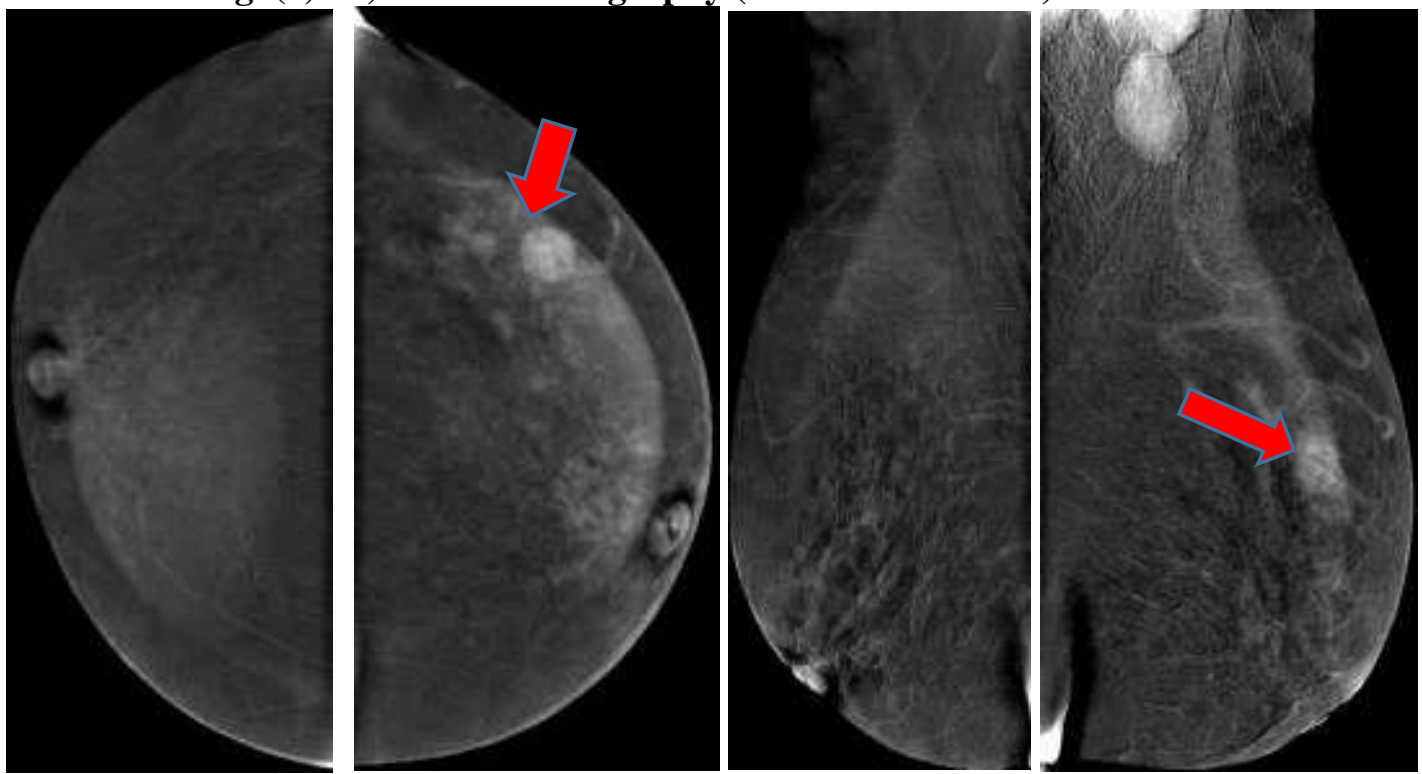

Fig. (8): B) Contrast enhanced spectral Mammography (CC \& MLO views).

\section{DISCUSSION}

In this study, the most frequent age category was that of $40-59$ years old $(56.7 \%)$, which coincides with the CDC ${ }^{(10)}$, statement that most breast cancers are diagnosed after the age of $50.66 .7 \%$ of the lumps detected by mammography were malignant, compared to $56.7 \%$ proved malignant by CEDM BIRADS that was similar to the final diagnosis. These results could be explained in, which presented the high sensitivity and specificity of the CEDM a finding and an explanation that agrees with many other clinical studies, which proved that CEDM is significantly more sensitive and specific than mammography alone and has sensitivity and specificity comparable to contrastenhanced breast MRI ${ }^{(11)}$. In addition, Tennant $\boldsymbol{e t}$ al. (12) reported that several studies have demonstrated consistent superiority of contrast-enhanced spectral mammography (CESM) when compared to full-field digital mammography (FFDM). Similarly Cheung $\boldsymbol{e t}$ al. (13) concluded that CESM provided additional information over mammography, with consistent improvement of cancer diagnosis especially in women with a dense breast parenchymal pattern.

Moreover Lobbes et al $^{\left({ }^{(4)}\right.}$ during his study of 113 CESM examinations performed in women recalled from the Dutch screening program found that MRI detected more reported ipsilateral additional foci of tumor cells in women diagnosed with a breast cancer (88\% of additional foci were detected using MRI compared to 56\% with CESM). However, CESM resulted in fewer false positives: 13 false positives were observed at MRI, compared to only two false 
positives using CESM and Dromain et al. ${ }^{(14)}$ who confirmed that the initial clinical results show that CESM has better diagnostic accuracy than mammography alone and mammography plus ultrasound. CESM showed a better lesion detection and size estimation than mammography in comparison to postoperative histology as the gold standard.

There was a highly significant association between mammography and CEDM end diagnosis, which is considered a good evidence on the very good sensitivity and specificity levels and its role in the accurate diagnosis of breast lumps that's considered very important professional practice to help in saving patients' lives. Cheung et al. (13) supported these findings as they found that CESM improved the cancer diagnosis by $21.2 \%$ in sensitivity $(71.5-92.7 \%$ ), by $16.1 \%$ in specificity $(51.8-67.9 \%)$ and by $19.8 \%$ in accuracy (65.9- 85.8\%) compared with mammography.

There was a notably high sensitivity and specificity levels $(100.0 \%, 92.86 \%)$ of the CEDM testing respectively. A result that's comparable to many other studies in this field such as the study of Mohamed et al. ${ }^{(15)}$, which revealed that malignant breast lesions uptake of contrast was significant with an overall sensitivity and specificity of the CEDM being $88.99 \%$ and $83.33 \%$ respectively. In addition, negative and positive probability ratios were 0.13 and 5.34 respectively.

Similarly Saraya et al. $^{\left({ }^{(16)}\right.}$ stated that contrastenhanced digital mammography proved to be more useful in the detection of the nature of the breast lesions compared to the FFDM and it also aids in the identification of the disease extent (multifocality and multicentricity) as well as in the axillary metastases. Further, it is beneficial in the guidance of core needle biopsy or excision because in positive cases lesion identification is much easier in the post contrast images in the high density breasts so it helps more in exact localization either for biopsy or wire localization for excision and when it is accessible it will be relatively less costly than the MR imaging technique. Also, Sudhir et al. ${ }^{(17)}$ study confirmed CEDM to have higher diagnostic accuracy than mammography alone or with ultrasound. Hence, CEDM can be used as a low-cost alternative to MRI as a problem-solving tool in regular clinical practice and therapeutic planning of breast cancer, which may include high-risk screening, dense breast evaluation, equivocal cases, microcalcifications, local staging, treatment response evaluation and post-treatment follow-up.

Moreover, Dromain et al. ${ }^{(14)}$ concluded that CEDM imaging may help improving the visibility of suspicious findings and their differentiation thanks to its depiction of tumor angiogenesis. CEDM has the potential to increase breast cancer detection rates, improve staging of breast cancers, and improve patient selection for biopsy. They also, added that the visibility of lesions with the addition of CEDM to mammography (MX) was considered to be superior to MX \pm US in more than $40 \%$ of cases, although some readers had little or no experience in interpreting CEDM images.

Besides, Moustafa et al. (18) reported that CESM showed high sensitivity regarding the identification of multiplicity reaching to $100 \%$ but with suboptimal specificity, $97.3 \%$ as some benign lesions could depict a significant enhancement such as inflammatory lesions, some benign tumors like fibroadenoma and, intra ductal papilloma. Osama $\boldsymbol{e t}$ al. (19) concluded that CESM is of special value in patients with dense breast tissue showing a greater diagnostic confidence and less operator dependency than mammography alone. This can be seen in a screening as well as non-screening context as it can help detect occult breast lesions in dense breasts. CEDM could be used as a "problem solving" technique in the cases with lesions of intermediate suspicion on mammography (BIRADS 3 or 4 lesions) for example areas of asymmetry with no complementary ultrasound findings. This can help decrease the number of unnecessary biopsies. Similarly the study of Lalji et al. (20) showed that CESM remains an excellent problem-solving tool for patients recalled from breast cancer screening, even when radiologists less experienced in CESM are reviewing the images.

ROC curve analysis based on the BI-RADS assessment for differentiating benign and malignant lesions in Sudhir et al. ${ }^{(21)}$ study revealed AUC of 0.896 for CESM, 0.841 for digital breast tomosynthesis (DBT) + ultrasound, 0.769 for DBT alone and 0.729 for synthesized, two-dimensional mammography (s2DMG).

In the same context, the results of Xing $\boldsymbol{e t ~ a l}$. (22) showed that CESM and MRI were comparable to the diagnostic efficacy of breast diseases and were even better than MRI in terms of specificity and diagnostic conformance rate. Moreover, in the prediction of tumor size, the difference between CESM and pathological results was not statistically significant. Therefore, we believe that CESM can well detect and diagnose breast diseases. On their study that was performed on large cohort of patients, Bozzini $\boldsymbol{e t}$ al. (23) confirmed that CESM provides additional information with consistent improvement of the cancer diagnosis in dense breasts and assessment in tumor size.

James and Tennant ${ }^{(24)}$ concluded that CESM is a much better imaging test than a "poor-man's" MRI. It should be considered as the primary mammographic examination replacing conventional mammography in selected patient groups. Used in this way, it can provide improved tumor detection, confidence of normality in women with a denser background pattern and supply more accurate tumor staging information at the woman's first attendance. Consequently, CESM has great potential as a first-line 
imaging technique in the symptomatic clinic or as a screening tool for women currently poorly served by conventional screening mammography.

\section{CONCLUSION}

Although mammography and ultrasound remain the primary diagnostic imaging modalities for the breast evaluation worldwide, several studies and this initial experience also proved that CEDM has higher diagnostic value that can enhance the process of early cancer diagnosis, postoperative follow up, and treatment plans. However, CEDM has a few limitations such as administration of iodinated contrast media with low risk of contrast reactions, theoretical risk from radiation exposure and low rates of false positive and false negative results.

\section{REFERENCES}

1. Klein S (2005): Evaluation of palpable breast masses. Am Fam Physician, 71(9):1731-1738.

2. Guray M, Sahin A (2006): Benign breast diseases: Classification, diagnosis, and management. Oncologist, 11(5):435-49.

3. Yu Y, Xiao Y, Cheng J et al. (2018): Breast lesion classification based on supersonic shear-wave elastography and automated lesion segmentation from B-mode ultrasound images. Comput Biol Med., 93:3146.

4. Lobbes M, Lalji U, Houwers J et al. (2014): Contrastenhanced spectral mammography in patients referred from the breast cancer screening programme. Eur Radiol., 24(7):1668-76.

5. Covington M, Pizzitola V, Lorans R et al. (2018): The future of contrast-enhanced mammography. American Journal of Roentgenology, 18:292-300.

6. Mokhtar O and Mahmoud S (2014): Can contrast enhanced mammography solve the problem of dense breast lesions? Egypt J Radiol Nucl Med., 45(3):104352.

7. Phillips J, Steinkeler J, Talati K et al. (2018): Workflow considerations for incorporation of contrastenhanced spectral mammography into a breast imaging practice. J Am Coll Radiol., 15(6):881-5.

8. Mori M, Akashi-Tanaka S, Suzuki S et al. (2017): Diagnostic accuracy of contrast-enhanced spectral mammography in comparison to conventional full-field digital mammography in a population of women with dense breasts. Breast Cancer, 24(1):104-10.

9. Jochelson M, Dershaw D, Sung J et al. (2013): Bilateral contrast-enhanced dual-energy digital mammography: Feasibility and comparison with conventional digital mammography and MR imaging in women with known breast carcinoma. Radiology, 266(3):743-51.

10. Centers for Disease Control and Prevention (2020): Division of Cancer Prevention and Control. What Does It Mean to Have Dense Breasts? https://www.cdc.gov/cancer/breast/basic_info/densebreasts.htm

11. Turnbull C (2021): Statistical approaches to interim monitoring of medical trials: a review and commentary. Statistical Science, 5(3): 299-317.
12. Tennant S, James J, Cornford E et al. (2016): Contrast-enhanced spectral mammography improves diagnostic accuracy in the symptomatic setting. Clin Radiol., 71(11):1148-55.

13. Cheung Y, Lin Y, Wan Y et al. (2014): Diagnostic performance of dual-energy contrast-enhanced subtracted mammography in dense breasts compared to mammography alone: interobserver blind-reading analysis. Eur Radiol., 24(10):2394-403.

14. Dromain C, Thibault F, Diekmann F et al. (2012): Dual-energy contrast-enhanced digital mammography: Initial clinical results of a multireader, multicase study. Breast Cancer Res., 14(3):94-99.

15. Mohamed Kamal R, Hussien Helal M, Wessam R et al. (2015): Contrast-enhanced spectral mammography: Impact of the qualitative morphology descriptors on the diagnosis of breast lesions. Eur J Radiol., 84(6):104955.

16. Saraya S, Adel L, Mahmoud A (2017): Indeterminate breast lesions: Can contrast enhanced digital mammography change our decisions? Egypt J Radiol Nucl Med., 48(2):547-52.

17. Sudhir R, Koppula V, Mandava A et al. (2021): Technique and clinical applications of dual-energy contrast-enhanced digital mammography (CEDM) in breast cancer evaluation: A pictorial essay. Diagnostic Interv Radiol., 27(1):28-36.

18. Moustafa A, Kamal E, Hassan M et al. (2018): The added value of contrast enhanced spectral mammography in identification of multiplicity of suspicious lesions in dense breast. Egypt J Radiol Nucl Med., 49(1):259-64.

19. Osama N, Hashem L, Kamal R et al. (2021): Does contrast-enhanced mammography have an impact on the detection of cancer in patients with risk of developing breast cancer? Egyptian Journal of Radiology and Nuclear Medicine, 52: 1-10.

20. Lalji U, Houben I, Prevos $\mathrm{R}$ et al. (2016): Contrastenhanced spectral mammography in recalls from the Dutch breast cancer screening program: validation of results in a large multireader, multicase study. Eur Radiol., 26(12):4371-9.

21. Sudhir R, Sannapareddy K, Potlapalli A et al. (2020): Diagnostic accuracy of contrast-enhanced digital mammography in breast cancer detection in comparison to tomosynthesis, synthetic 2D mammography and tomosynthesis combined with ultrasound in women with dense breast. Br J Radiol., 94(1118):20201046.

22. Xing D, Lv Y, Sun B et al. (2019): Diagnostic value of contrast-enhanced spectral mammography in comparison to magnetic resonance imaging in breast lesions. J Comput Assist Tomogr., 43(2): 245-251.

23. Bozzini A, Nicosia L, Pruneri G et al. (2020): Clinical performance of contrast-enhanced spectral mammography in pre-surgical evaluation of breast malignant lesions in dense breasts: a single center study. Breast Cancer Res Treat., 184(3):723-31.

24. James J, Tennant S (2018): Contrast-enhanced spectral mammography (CESM). Clinical Radiology, 73: 715-23. 